\title{
Chinese Herbal Formulas Si-Wu-Tang and Er-Miao-San Synergistically Ameliorated Hyperuricemia and Renal Impairment in Rats Induced by Adenine and Potassium Oxonate
}

\author{
Yongping Guo Qian Jiang ${ }^{\mathrm{b}}$ Dingkun Guia Niansong Wang ${ }^{\mathrm{a}}$ \\ aDepartment of Nephrology and Rheumatology, Shanghai Jiaotong University Affiliated Sixth People's \\ Hospital, Shanghai, bDepartment of Nephrology, East Hospital, Tongji University, Shanghai, PR China
}

\section{Key Words}

Chinese herbal formula $・$ Si-Wu-Tang $•$ Er-Miao-San $・$ Hyperuricemia $•$ OAT1 $・$ OAT3

\begin{abstract}
Background/Aims: Hyperuricemia is an independent risk factor for chronic kidney disease and cardiovascular disease. Here, we examined the combined protective effects of Chinese herbal formula Si-Wu-Tang and Er-Miao-San on hyperuricemia and renal impairment in rats. Methods: Rats were randomly divided into normal rats, hyperuricemic rats, and hyperuricemic rats orally administrated with benzbromarone $\left(4.5 \mathrm{mg} \cdot \mathrm{kg}^{-1} \cdot \mathrm{d}^{-1}\right)$, Si-Wu-Tang $\left(3.78 \mathrm{~g} \cdot \mathrm{kg}^{-1} \cdot \mathrm{d}^{-1}\right)$ and Si-Wu-Tang plus Er-Miao-San $\left(6.48 \mathrm{~g} \cdot \mathrm{kg}^{-1} \cdot \mathrm{d}^{-1}\right)$ for 4 weeks. Hyperuricemic rats were orally gavaged with adenine $\left(0.1 \mathrm{~g} \cdot \mathrm{kg}^{-1} \cdot \mathrm{d}^{-1}\right)$ and potassium oxonate $\left(1.5 \mathrm{~g} \cdot \mathrm{kg}^{-1} \cdot \mathrm{d}^{-1}\right)$ daily for 4 weeks. Serum uric acid, creatinine, total cholesterol (TCH), triglyceride and blood urea nitrogen (BUN) concentrations, as well as urinary uric acid and microalbuminuria were measured weekly. Serum xanthine oxidase (XOD) activity and renal histopathology were also evaluated. The renal expression of organic anion transporter 1 (OAT1) and organic anion transporter 3 (OAT3) was detected by western blot. Results: Si-Wu-Tang plus Er-Miao-San lowered serum uric acid, creatinine, triglyceride and BUN levels to a greater degree than did Si-Wu-Tang alone. Si-WuTang plus Er-Miao-San ameliorated microalbuminuria and renal histopathology, as well as decreased serum TCH concentration and XOD activity in hyperuricemic rats. Combination of Si-Wu-Tang and Er-Miao-San also led to a greater increase in OAT1 and OAT3 expression than did Siwutang alone. Conclusion: Si-Wu-Tang and Er-Miao-San synergistically ameliorated hyperuricemia and renal impairment in rats through upregulation of OAT1 and OAT3.
\end{abstract}

Copyright $(2015$ S. Karger AG, Basel

\section{Introduction}

The overall prevalence of chronic kidney disease (CKD) in China was 10.8\% and there were about 119.5 million Chinese patients with CKD [1]. Hyperuricemia is an independent

Dingkun Gui and Niansong Wang

KARGER 125
Department of Nephrology and Rheumatology, Shanghai Jiaotong University Affiliated Sixth People's Hospital, 600 Yishan Road, Shanghai 200233, (China)

E-Mail guidingkun@sina.com.cn and E-Mail wangniansong2012@163.com 
risk factor for the development of CKD [2] and independently associated with kidney damage $[1,3]$. Uric acid is also an independent risk factor for cardiovascular, ischemic stroke and all-cause mortality in the general population [4,5]. Despite advances in the use of antihyperuricemic agents for the treatment of hyperuricaemia and gout, allopurinol, as an inhibitor of xanthine oxidase (XOD), could cause severe hypersensitivity and agranulocytosis, and aggravate renal toxicity by impairing pyrimidine metabolism [6-9]. These adverse side effects limited its clinical use. Thus, there is a pressing need for the development of new hypouricemic agents with greater effectiveness and safety, especially herbal medicine [10, $11]$.

Herbal medicines have traditionally played an important role in treating and preventing human diseases in China for thousands of years. Si-Wu-Tang, which is composed of Rehmannia glutinosa, Angelica sinensis, Radix Paeoniae Alba and Rhizoma Chuanxiong, was originally listed in the Prescriptions from the Great Peace Imperial Grace Pharmacy and has been used as a basic formula in traditional Chinese medicine since the 12th century. SiWu-Tang is widely used for its action as a blood-tonifying (buxie) decoction [12]. Aqueous extract $\mathrm{Si}$-Wu-Tang was reported to have antipruritic and antiinflammatory effects in mice [13]. Another herbal formula Er-Miao-San, which is composed of the Cortex Phellodendri Chinensis and Atractylodes Lancea, has been used in traditional medicine for a long time. According to Dan-Xi-Xin-Fa (Zhu Zhenhen, Yuan dynasty: a Chinese medicine book named Danxi's Experiences in Medicine), Er-Miao-San was descried to treat acute gout through eliminating heat and excreting dampness in terms of traditional Chinese medicine. Er-MiaoSan was also recorded in State Pharmacopoeia of People's Republic of China at all times for the successful treatment of gout and hyperuricemia. Previous study demonstrated that Er-Miao-Wan decreased serum uric acid level and inhibited liver xanthine dehydrogenase and xanthine oxidase in mice [14]. However, the effects of Si-Wu-Tang and Er-Miao-San on serum uric acid and renal injury in hyperuricemic rats have not been investigated yet. This study aimed to investigate the combined protective effects of Si-Wu-Tang and Er-Miao-San on hyperuricemia and renal impairment in rats induced by adenine and potassium oxonate, and then provide a novel therapeutic approach for the treatment of uric acid nephropathy.

\section{Materials and Methods}

\section{Plant material}

The ingredients of Si-Wu-Tang included $12 \mathrm{~g}$ of Rehmannia glutinosa, $10 \mathrm{~g}$ of Angelica sinensis, 12 g of Radix Paeoniae Alba and $8 \mathrm{~g}$ of Rhizoma Chuanxiong. Er-Miao-San was composed of $15 \mathrm{~g}$ of Cortex Phellodendri Chinensis and $15 \mathrm{~g}$ of Atractylodes Lancea. All the herbs used in this study were purchased from Chinese Herbal Medicine Dispensary of Shanghai East Hospital.

Preparation of the aqueous extract

An extract of $\mathrm{Si}$-Wu-Tang was prepared by decocting the dried prescription of herbs (12 $\mathrm{g}$ of Rehmannia glutinosa, $10 \mathrm{~g}$ of Angelica sinensis, $12 \mathrm{~g}$ of Radix Paeoniae Alba and $8 \mathrm{~g}$ of Rhizoma Chuanxiong) with boiling water for $1 \mathrm{~h}$ and extracted three times. The obtained suspension was separated by filtration and condensed to the concentration of $1.556 \mathrm{~g} / \mathrm{ml}$ solution and then stored at $4^{\circ} \mathrm{C}$ before administration. The mixtures of Si-Wu-Tang and Er-Miao-San, including $12 \mathrm{~g}$ of Rehmannia glutinosa, $10 \mathrm{~g}$ of Angelica sinensis, $12 \mathrm{~g}$ of Radix Paeoniae Alba and $8 \mathrm{~g}$ of Rhizoma Chuanxiong, $15 \mathrm{~g}$ of Cortex Phellodendri Chinensis and 15 $\mathrm{g}$ of Atractylodes Lancea, were boiled in 10 volumes of water (v/w) for $1 \mathrm{~h}$ and extracted three times. The suspension was then separated by filtration and condensed to the concentration of $0.706 \mathrm{~g} / \mathrm{ml}$ solution and then stored at $4{ }^{\circ} \mathrm{C}$ before use. Si-Wu-Tang was administrated to rats at $3.78 \mathrm{~g} \cdot \mathrm{kg}^{-1} \cdot \mathrm{d}^{-1}$ and Si-Wu-Tang plus Er-Miao-San was given to rats at $6.48 \mathrm{~g} \cdot \mathrm{kg}^{-1} \cdot \mathrm{d}^{-1}$.

Reagents and drugs

Adenine was purchased from Sinopharm Chemical Reagent Co.Ltd, Shanghai, China and potassium oxonate was purchased from Shandong Zhongke Taidou Chemical Co. Ltd, Jinan, China. Adenine and 


\section{Cellular Physiology Cell Physiol Biochem 2015;37:1491-1502

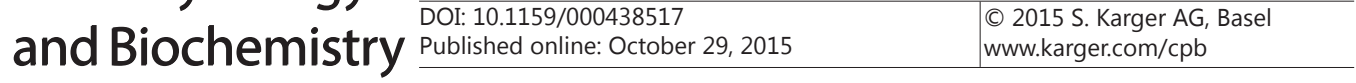

potassium oxonate were suspended in distilled water at the concentration of $80 \mathrm{~g} / \mathrm{L}$. Benzbromarone, purchased from from Heumann Pharma GmbH, Germany, was suspended in distilled water and then stored at $4^{\circ} \mathrm{C}$ before use. Benzbromarone was given to rats at $4.5 \mathrm{mg} \cdot \mathrm{kg}^{-1} \cdot \mathrm{d}^{-1}$.

\section{Animal study}

All the animal procedures were performed in accordance with the "Guide for the Care and Use of Laboratory Animals" published by the National Institutes of Health. All the animal protocols were approved by the Animal Ethics Committee of Shanghai University of Traditional Chinese Medicine, Shanghai, China. Healthy male Sprague-Dawley rats weighing 180 to 200 g, purchased from Experimental Animal Center, Shanghai University of Traditional Chinese Medicine, Shanghai, China., were housed in an air-conditioned room at $22 \pm 2{ }^{\circ} \mathrm{C}$ on a 12:12-h light-dark cycle. Animals were fed a standard diet and given water ad libitum. After 1 week of acclimation, rats were randomly divided into five groups ( $\mathrm{n}=10$ /each group): (1) normal rats used as control (NC); (2) hyperuricemic rats (HU); (3) hyperuricemic rats treated with benzbromarone (BM); (4) hyperuricemic rats treated with Si-Wu-Tang (SW) and (5) hyperuricemic rats treated with SiWu-Tang plus Er-Miao-San(SW + EM). Rats were treated for 4 weeks. Hyperuricemia was induced in rats by orally garage with adenine $\left(0.1 \mathrm{~g} \cdot \mathrm{kg}^{-1} \cdot \mathrm{d}^{-1}\right)$ and potassium oxonate $\left(1.5 \mathrm{~g} \cdot \mathrm{kg}^{-1} \cdot \mathrm{d}^{-1}\right)$ daily for 4 weeks. The normal control and hyperuricemic control rats received the equal volume of saline within the same time. Rats were kept in individual metabolic cages for $24 \mathrm{~h}$ urine collection. Urine was centrifuged at $800 \mathrm{~g}$ for 10 $\min$ at $25^{\circ} \mathrm{C}$. Whole urine was stored at $-70^{\circ} \mathrm{C}$ and thawed just before use. Urine microalbumin, creatinine and uric acid concentrations were detected by an automatic biochemistry analyzer (Hitachi Model 7600, Japan). Rats were then anesthetized with pentobarbital sodium and the blood samples were taken through the abdominal aorta for measuring XOD activity, as well as biochemical parameters, including blood urea nitrogen (BUN), creatinine ( $\mathrm{Cr}$ ), total cholesterol (TCH), and triglyceride (TG) concentrations by an automatic biochemistry analyzer (Hitachi Model 7600, Japan). Serum uric acid, creatinine, TCH, TG, BUN concentrations, as well as urinary uric acid and microalbuminuria were measured weekly. Animals were then killed and the kidneys were harvested immediately. The cortex from transversely bisected left kidneys was snap-frozen in liquid nitrogen and stored at $-70^{\circ} \mathrm{C}$ for protein extraction.

\section{Light microscopy}

That of the right kidneys was fixed with $10 \%$ buffered formalin and embedded in paraffin for histological evaluation. The kidneys were cut into $3 \mu \mathrm{m}$ sections and stained with hematoxylin and eosin. The sections were also stained routinely with Masson's trichrome. The sections were then examined by light microscopy in a blind fashion.

Measurement of serum xanthine oxidase (XOD) activity

The activity of XOD in serum was determined using the assay kits (Nanjing Jiancheng Bioengineering Institute, Nanjing, China), according to the manufacturer's instruction. Serum XOD activity was determined by UV spectrophotometer (Shimadzu Corporation, Japan).

\section{Western blotting}

Tissue samples from renal cortex were homogenized and lysed. The lysates were clarified by centrifugation at 12,000 x g for $15 \mathrm{~min}$. Protein estimation was performed by Coomassie brilliant blue. The tissue protein was separated by sodium dodecyl sulfate (SDS)/polyacrylamide gel electrophoresis and transferred to a polyvinylidene difluoride membrane (Millopore, USA). The membranes were blocked for $1 \mathrm{~h}$ at room temperature with PBS containing 5\% skim milk. Membranes were then incubated overnight with OAT1 and OAT3 antibodies (ABBIOTEC, San Diego, USA); Negative controls were performed without primary antibody. After washing, the secondary antibody was added and incubated $1 \mathrm{~h}$ at room temperature. After membranes were washed with TBS containing $0.05 \%$ Tween 20 , the blots were visualized using enhanced chemiluminescence (ECL) immunoblot detection kits (Millopore, USA). Equality of loading was ensured by using a monoclonal antibody to $\beta$-actin (Beyotime Institute of Biotechnology, Shanghai, China). Densitometric quantitation was performed using a Bio-Rad VersaDoc imaging system model 5000 with Bio-Rad Quantity One software. Protein expression was quantified as the ratio of specific band to $\beta$-actin. Relative protein expression was described as the fold change from the normal control group.

\section{KARGER}


Statistical analysis

Statistical analyses were conducted by using SPSS 13.0 software. All data were expressed as means \pm standard deviation (SD). The significance of differences among experimental groups was determined by analysis of variance (ANOVA) followed by Dunnett's multiple range test. Differences among groups were evaluated by nonparametric test when necessary. A $P$ value $<0.05$ was considered statistically significant.

\section{Results}

Effects of Si-Wu-Tang and Si-Wu-Tang plus Er-Miao-San on serum and urinary uric acid levels in hyperuricemic rats

The levels of serum and urinary uric acid were shown in Fig. 1. The hyperuricemic rats showed a significant increase in serum uric acid concentration after one week of oral administration of adenine and potassium oxonate when compared with the normal control rats $(P<0.05)$. Treatment with Si-Wu-Tang plus Er-Miao-San for 4 weeks lowered serum uric acid level to a greater degree than did Si-Wu-Tang alone (Fig. 1A). Benzbromarone also decreased the serum uric acid level at the end of 4 weeks of treatment. However, Si-Wu-Tang plus Er-Miao-San induced a greater decrease in serum uric acid level than did benzbromarone (Fig. 1A). Moreover, treatment with benzbromarone for 4 weeks significantly promoted the urinary excretion of uric acid, while neither Si-Wu-Tang nor Si-Wu-Tang plus Er-Miao-San increased the urinary excretion of uric acid (Fig. 1B) in hyperuricemic rats. The above results demonstrated that the hypouricemic effect of Si-Wu-Tang plus Er-Miao-San was greater than that of Si-Wu-Tang alone or benzbromarone.

Effects of Si-Wu-Tang and Si-Wu-Tang plus Er-Miao-San on serum creatinine and BUN levels in hyperuricemic rats

At end of 2 weeks of treatment, Si-Wu-Tang plus Er-Miao-San significantly lowered the serum creatinine level when compared with the untreated hyperuricemic rats. SiWu-Tang plus Er-Miao-San decreased serum creatinine level to a greater degree than did

Fig. 1. Effects of Si-Wu-Tang and ErMiao-San on serum uric acid (A) and urinary uric acid (B) levels in hyperuricemic rats. NC, normal rats used as control; HU, hyperuricemic rats; BM, hyperuricemic rats treated with benzbromarone at $4.5 \mathrm{mg} \cdot \mathrm{kg}^{-1} \cdot \mathrm{d}^{-1} ; \mathrm{SW}$, hyperuricemic rats treated with Si-WuTang at $3.78 \mathrm{~g} \cdot \mathrm{kg}^{-}$ ${ }^{1} \cdot \mathrm{d}^{-1} ; \mathrm{SW}+\mathrm{EM}$, hyperuricemic rats treated with Si-Wu-Tang plus Er-Miao-San at $6.48 \mathrm{~g} \cdot \mathrm{kg}^{-1} \cdot \mathrm{d}^{-1}$. Rats
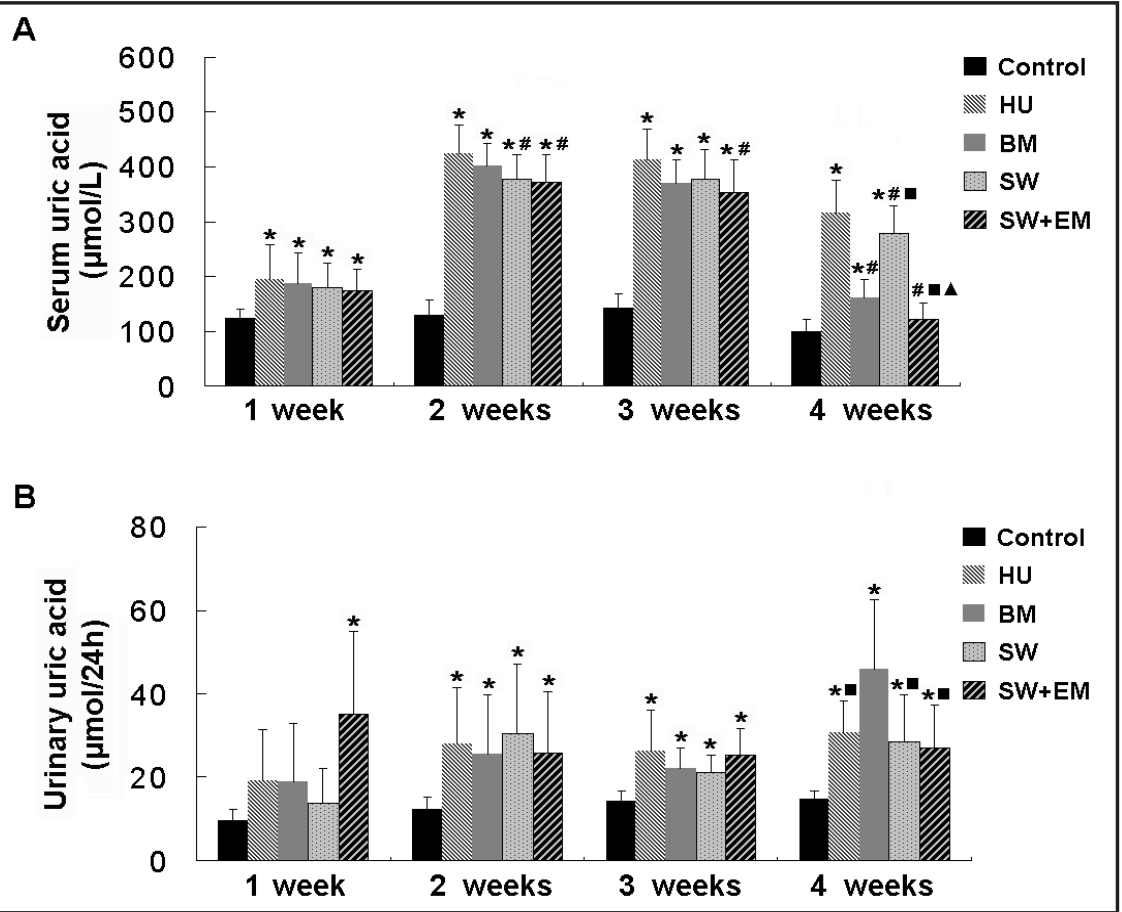

were treated for 4 weeks. Results are expressed as the means $\pm \mathrm{SD}(\mathrm{n}=10){ }^{*} P<0.05$ vs NC group; \# $P<0.05$ vs HU group; $\bullet P<0.05$ vs BM group; $\stackrel{\wedge}{ } P<0.05$ vs $S W$ group. 
Fig. 2. Effects of SiWu-Tang and Er-Miao-San on serum creatinine (A) and blood urea nitrogen (B) levels in hyperuricemic rats. $\mathrm{NC}$, normal rats used as control; $\mathrm{HU}$, hyperuricemic rats; BM; hyperuricemic rats treated with benzbromarone at $4.5 \mathrm{mg} \cdot \mathrm{kg}^{-1} \cdot \mathrm{d}^{-1} ; \quad \mathrm{SW}$, hyperuricemic rats treated with Si-WuTang at $3.78 \mathrm{~g} \cdot \mathrm{kg}^{-1} \cdot \mathrm{d}^{-1}$; SW+EM, hyperuricemic rats treated with Si-Wu-Tang plus Er-Miao-San at 6.48 $\mathrm{g} \cdot \mathrm{kg}^{-1} \cdot \mathrm{d}^{-1}$. Rats were treated for 4 weeks. Results are expressed

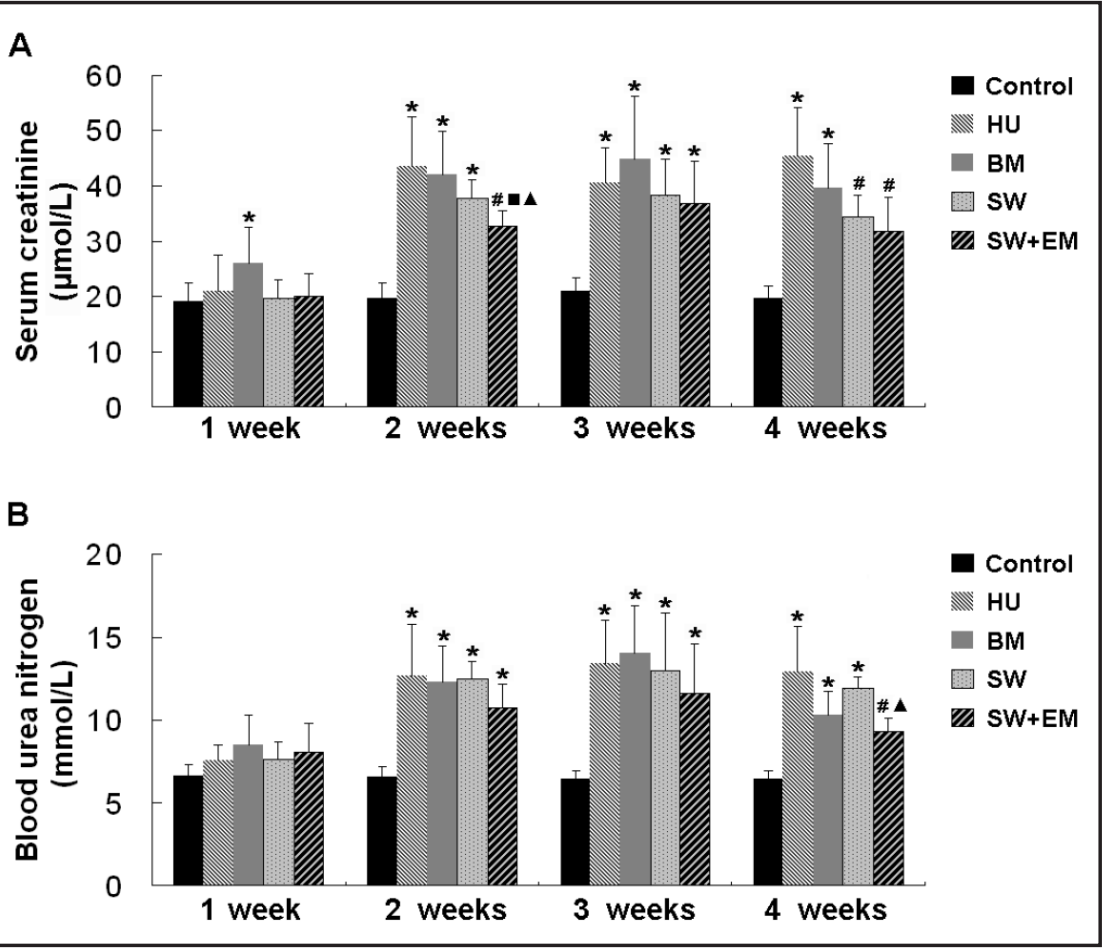
as the means $\pm \mathrm{SD}(\mathrm{n}=10){ }^{*} P<0.05$ vs NC group; ${ }^{\#} P<0.05$ vs HU group; ${ }^{\boldsymbol{}} P<0.05$ vs BM group; ${ }^{\boldsymbol{\Delta}} P<0.05$ vs SW group.

Fig. 3. Effects of Si-Wu-Tang and ErMiao-San on serum total cholesterol $(\mathrm{A})$ and triglyceride (B) concentrations in hyperuricemic rats. $\mathrm{NC}$, normal rats used as control; $\mathrm{HU}$, hyperuricemic rats; $\mathrm{BM}$; hyperuricemic rats treated with benzbromarone at $4.5 \mathrm{mg} \cdot \mathrm{kg}$ ${ }^{1} \cdot \mathrm{d}^{-1}$; SW, hyperuricemic rats treated with Si-Wu-Tang at 3.78 $\mathrm{g} \cdot \mathrm{kg}^{-1} \cdot \mathrm{d}^{-1} ; \quad \mathrm{SW}+\mathrm{EM}$, hyperuricemic rats treated with $\mathrm{Si}-\mathrm{Wu}$ Tang plus Er-MiaoSan at $6.48 \mathrm{~g} \cdot \mathrm{kg}^{-1} \cdot \mathrm{d}^{-1}$. Rats were treated for

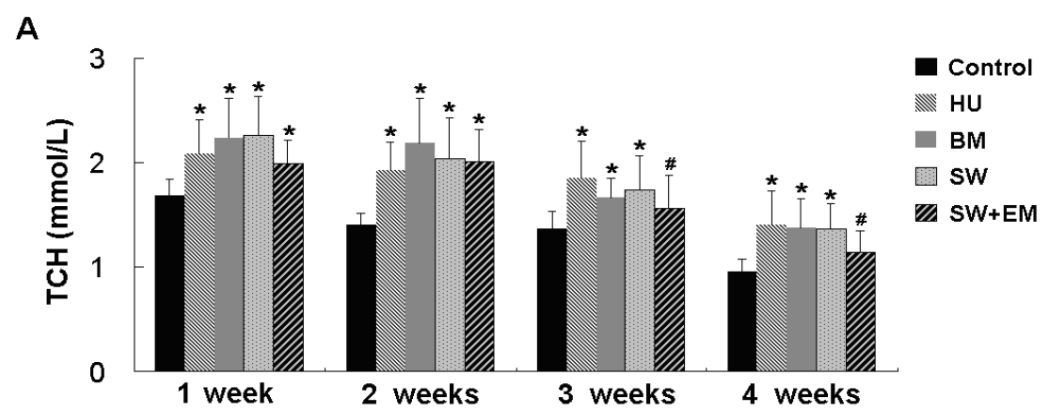

B

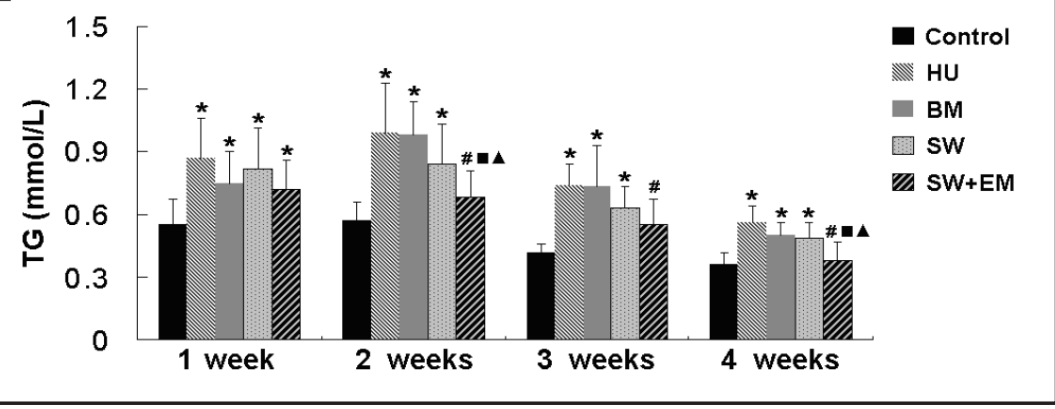

4 weeks. TCH, total cholesterol; TG, triglyceride. Results are expressed as the means $\pm \mathrm{SD}(\mathrm{n}=10) .{ }^{*} P<0.05$ vs NC group; ${ }^{\#} P<0.05$ vs HU group; $\boldsymbol{*} P<0.05$ vs BM group; ${ }^{\wedge} P<0.05$ vs SW group.

Si-Wu-Tang alone. At end of 4 weeks of treatment, both Si-Wu-Tang and Si-Wu-Tang plus Er-Miao-San significantly decreased the serum creatinine level when compared with the untreated hyperuricemic rats (Fig. 2A). Moreover, Si-Wu-Tang plus Er-Miao-San significantly 
Fig. 4. Effects of SiWu-Tang and Er-Miao-San on microalbuminuria (A) and serum xanthine oxidase activity (B) in hyperuricemic rats. $\mathrm{NC}$, normal rats used as control; HU, hyperuricemic rats; $\mathrm{BM}$; hyperuricemic rats treated with benzbromarone at $4.5 \mathrm{mg} \cdot \mathrm{kg}$ ${ }^{1} \cdot \mathrm{d}^{-1} ; \mathrm{SW}$, hyperuricemic rats treated with Si-Wu-Tang at 3.78 $\mathrm{g} \cdot \mathrm{kg}^{-1} \cdot \mathrm{d}^{-1} ; \mathrm{SW}+\mathrm{EM}, \mathrm{hy}-$ peruricemic rats treated with Si-Wu-Tang plus Er-Miao-San at

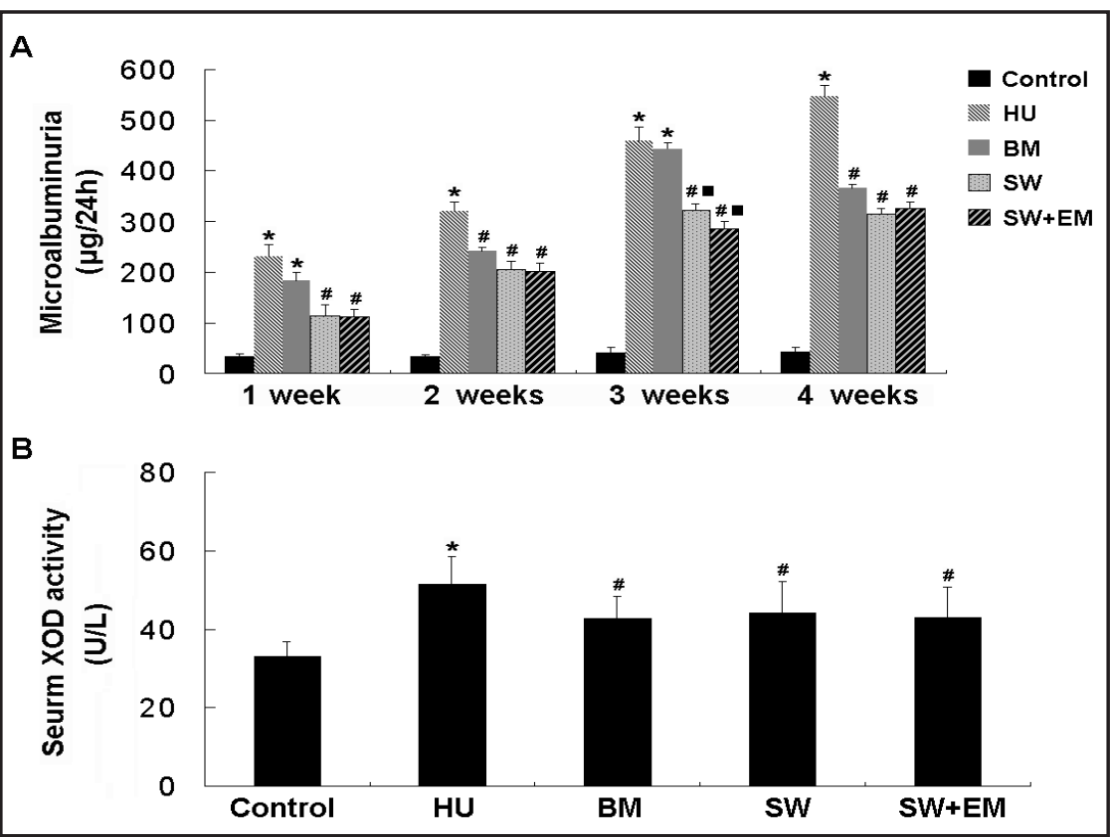
$6.48 \mathrm{~g} \cdot \mathrm{kg}^{-1} \cdot \mathrm{d}^{-1}$. XOD, xanthine oxidase; Rats were treated for 4 weeks. Results are expressed as the means \pm SD $(\mathrm{n}=10) .{ }^{*} P<0.05$ vs NC group; ${ }^{\#} P<0.05$ vs HU group; $P<0.05$ vs BM group.

lowered the BUN level at end of 4 weeks of treatment when compared with the untreated hyperuricemic rats, while Si-Wu-Tang plus Er-Miao-San had a substantial greater effect on decreasing BUN level than that of Si-Wu-Tang alone (Fig. 2B). The above results indicated that Si-Wu-Tang and Er-Miao-San synergistically ameliorated renal impairment in hyperuricemic rats.

Effects of Si-Wu-Tang and Si-Wu-Tang plus Er-Miao-San on serum level of TCH and TG in hyperuricemic rats

Si-Wu-Tang plus Er-Miao-San significantly reduced the serum level of TCH when compared with the untreated hyperuricemic rats, which was evident in as little as 3 weeks after treatment and reached the peak effect at the end of 4 weeks of treatment (Fig. 3A). Treatment with Si-Wu-Tang plus Er-Miao-San decreased the serum level of TG to a greater degree than did Si-Wu-Tang alone. This effect was evident at the end of 2 week of treatment and reached the peak effect at the end of 4 weeks of treatment (Fig. 3B).Thus, Si-Wu-Tang plus Er-Miao-San had a substantial greater effect on decreasing TG level than that of Si-WuTang alone.

Effects of Si-Wu-Tang and Si-Wu-Tang plus Er-Miao-San on microalbuminuria and serum $X O D$ activity in hyperuricemic rats

Compared with the normal control rats, hyperuricemic rats exhibited elevated urinary albumin excretion (expressed as the $\mu \mathrm{g} / 24 \mathrm{~h}$ ). Both Si-Wu-Tang and Si-Wu-Tang plus Er-MiaoSan significantly alleviated microalbuminuria in hyperuricemic rats (Fig. 4A). This effect was time-dependent, which was evident in as little as 1 week of treatment. Treatment with SiWu-Tang plus Er-Miao-San for 3 weeks decreased the microalbuminuria in hyperuricemic rats to a greater degree than did benzbromarone, however, no difference in urinary albumin excretion was observed between Si-Wu-Tang treated and Si-Wu-Tang plus Er-Miao-San treated hyperuricemic rats (Fig. 4A). Moreover, hyperuricemic rats showed elevated serum activity of XOD, which was reversed by Si-Wu-Tang, Si-Wu-Tang plus Er-Miao-San and benzbromarone (Fig. 4B). Therefore, Si-Wu-Tang plus Er-Miao-San significantly lowered microalbuminuria and serum XOD activity in hyperuricemic rats. 
Fig. 5. Effects of Si-Wu-Tang and Er-Miao-San on renal histopathology stained with hematoxylin and eosin in hyperuricemic rats. $\mathrm{NC}$, normal rats used as control; HU, hyperuricemic rats; $\mathrm{BM}$; hyperuricemic rats treated with benzbromarone at $4.5 \mathrm{mg} \cdot \mathrm{kg}^{-1} \cdot \mathrm{d}^{-1}$; SW, hyperuricemic rats treated with Si-Wu-Tang at 3.78 $\mathrm{g} \cdot \mathrm{kg}^{-1} \cdot \mathrm{d}^{-1} ; \mathrm{SW}+\mathrm{EM}$, hyperuricemic rats treated with $\mathrm{Si}-$ Wu-Tang plus Er-Miao-San at $6.48 \mathrm{~g} \cdot \mathrm{kg}^{-1} \cdot \mathrm{d}^{-1}$. Rats were treated for 4 weeks.

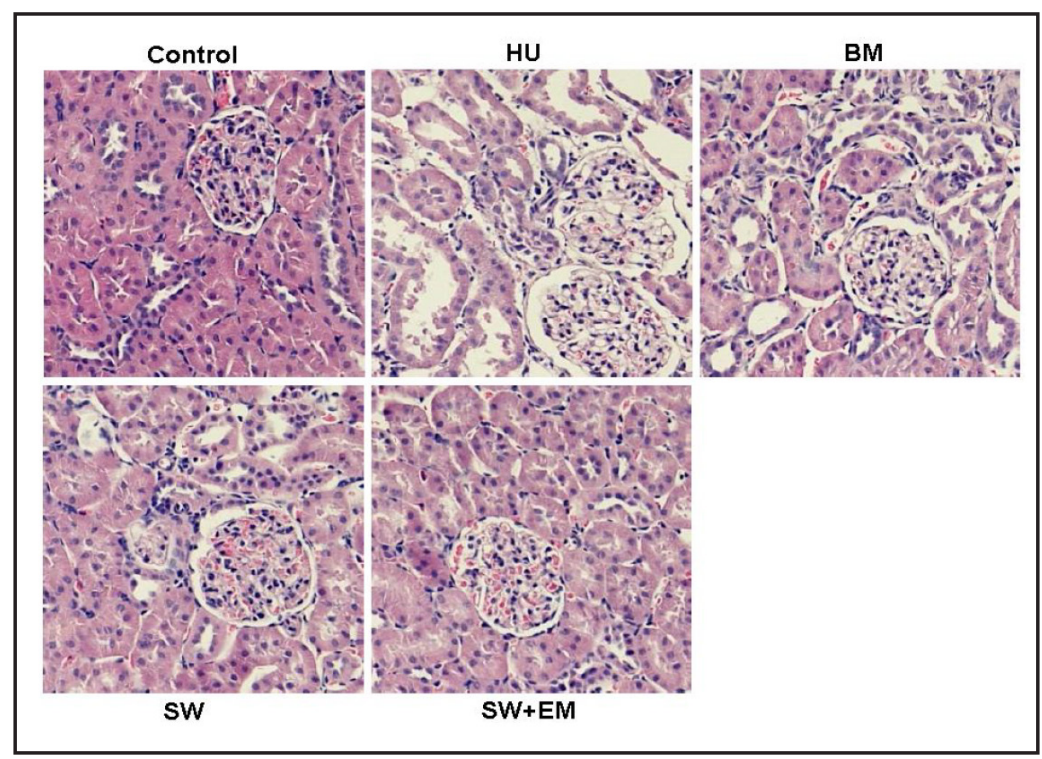

Fig. 6. Effects of Si-Wu-Tang and Er-Miao-San on renal histopathology stained with Masson's trichrome in hyperuricemic rats. $\mathrm{NC}$, normal rats used as control; HU, hyperuricemic rats; BM; hyperuricemic rats treated with benzbromarone at $4.5 \mathrm{mg} \cdot \mathrm{kg}^{-}$ ${ }^{1} \cdot \mathrm{d}^{-1}$; SW, hyperuricemic rats treated with Si-Wu-Tang at $3.78 \mathrm{~g} \cdot \mathrm{kg}^{-1} \cdot \mathrm{d}^{-1} ; \mathrm{SW}+\mathrm{EM}$, hyperuricemic rats treated with Si-Wu-Tang plus Er-Miao-San at $6.48 \mathrm{~g} \cdot \mathrm{kg}^{-1} \cdot \mathrm{d}^{-1}$. Rats were treated for 4 weeks.

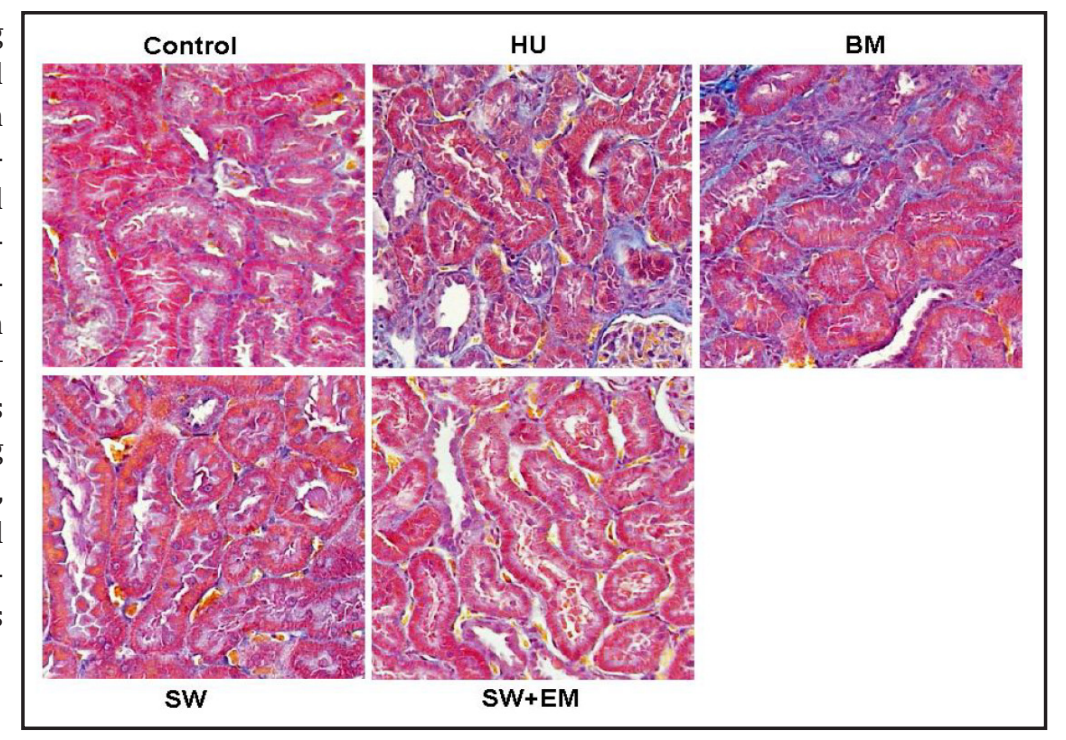

Effects of Si-Wu-Tang and Si-Wu-Tang plus Er-Miao-San on renal histopathology in hyperuricemic rats

At 4 weeks after adenine and potassium oxonate adminstration, the hyperuricemic rats showed vacuole in renal tubular epithelial cells, tubular dilation and inflammatory cell infiltration in renal tubular. These kidney pathological changes were ameliorated by the treatment of Si-Wu-Tang, Si-Wu-Tang plus Er-Miao-San and benzbromarone (Fig. 5). As shown in Masson's trichrome staining, the hyperuricemic rats developed tubular dilatation and expansive interstitial fibrosis when compared with the normal control rats. However, both Si-Wu-Tang and Si-Wu-Tang plus Er-Miao-San alleviated the development of renal interstitial fibrosis in hyperuricemic rats (Fig. 6).

Effects of Si-Wu-Tang and Si-Wu-Tang plus Er-Miao-San on OAT1 and OAT3 expression in hyperuricemic rats

Compared with the normal control rats, the hyperuricemic rats showed reduced expression of OAT1 and OAT3 (Fig. 7) detected by western blot. However, Si-Wu-Tang plus Er-Miao-San significantly increased renal expression of OAT1 (Fig. 7A) and OAT3 (Fig. 7B) in hyperuricemic rats. Quantitative analysis indicated that the OAT1 and OAT3 protein levels

\section{KARGER}




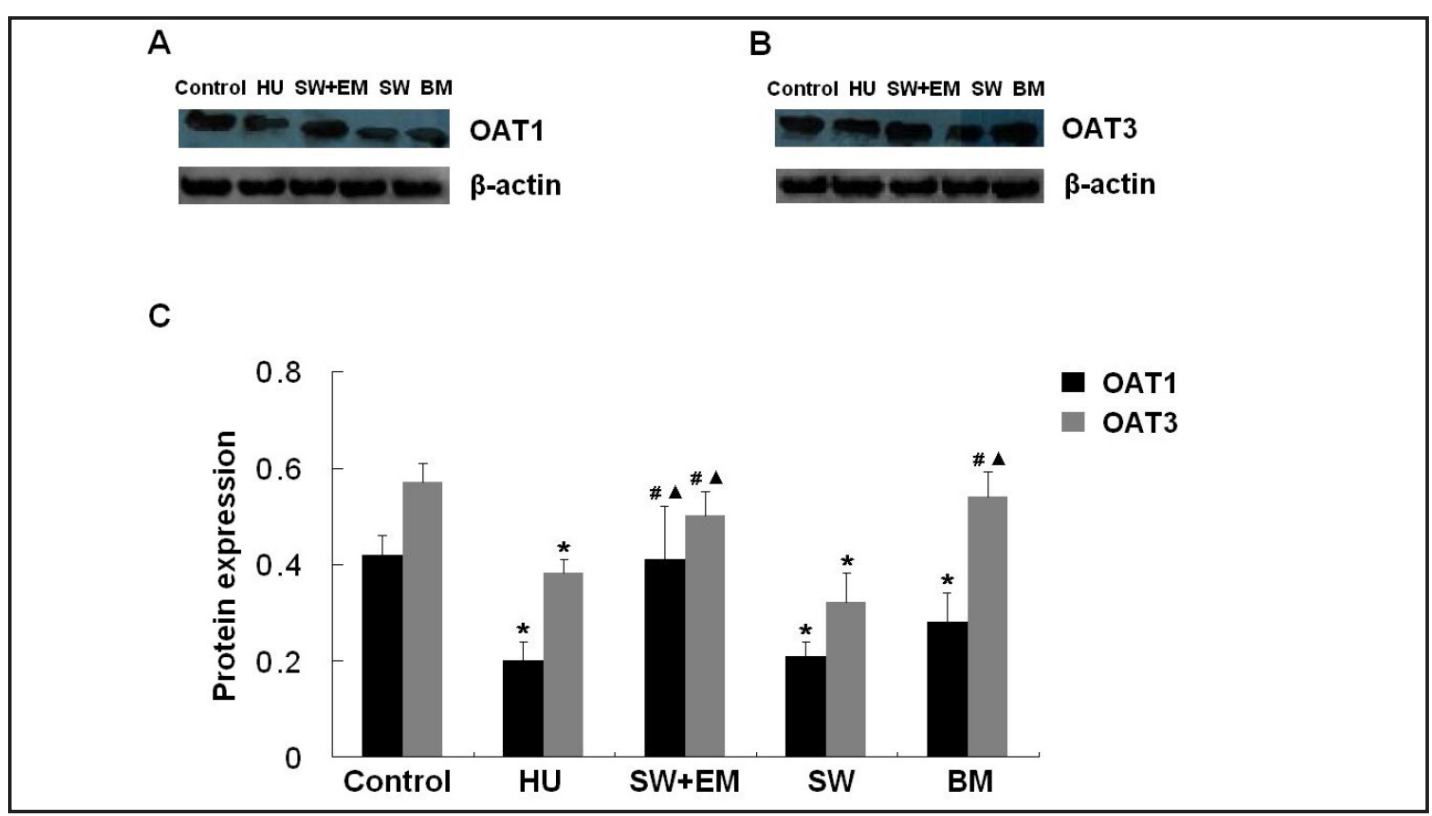

Fig. 7. Effects of Si-Wu-Tang and Er-Miao-San on OAT1 and OAT3 protein expression in hyperuricemic kidneys examined by Western blotting. NC, normal rats used as control; HU, hyperuricemic rats; BM; hyperuricemic rats treated with benzbromarone at $4.5 \mathrm{mg} \cdot \mathrm{kg}^{-1} \cdot \mathrm{d}^{-1} ; \mathrm{SW}$, hyperuricemic rats treated with $\mathrm{Si}-\mathrm{Wu}$-Tang at $3.78 \mathrm{~g} \cdot \mathrm{kg}^{-1} \cdot \mathrm{d}^{-1}$; SW+EM, hyperuricemic rats treated with Si-Wu-Tang plus Er-Miao-San at $6.48 \mathrm{~g} \cdot \mathrm{kg}^{-1} \cdot \mathrm{d}^{-1}$. OAT1, organic anion transporter 1; OAT3, organic anion transporter 3. Rats were treated for 4 weeks. Results are expressed as the means \pm SD. ${ }^{*} P<0.05$ vs NC group; ${ }^{\#} P<0.05$ vs HU group; ${ }^{\wedge} P<0.05$ vs SW group.

were decreased in hyperuricemic rats, which were reversed by Si-Wu-Tang plus Er-Miao-San (Fig. 7C).

\section{Discussion}

It was reported that gout, a common metabolic disorder in human, afflicted more than 2 million men and women in the United States [15]. Gout is also rapidly increasing in China due to recent changes in dietary habits [16]. Therapeutic drugs for gout treatment include the use of non-steroid anti-inflammtory drugs, which temporarily alleviate the symptoms, and of $\mathrm{XDH}$ and XO inhibitors, which inhibit the synthesis of uric acid from purines. However, these drugs have side effects and are not be sufficient to completely delay disease progression. Thus, there are high priorities to develop novel and effective therapeutic strategies to treat or prevent hyperuricemia. The novel finding of this study was that the Chinese herbal formulas Si-Wu-Tang and Er-Miao-San synergistically ameliorated hyperuricemia and renal impairment in rats, which was involved in upregulation of OAT1 and OAT3 expression. This conclusion was based upon the following findings: (i) Si-Wu-Tang plus Er-Miao-San lowered serum uric acid, creatinine and BUN levels to a greater degree than did Si-Wu-Tang alone. (ii) Si-Wu-Tang plus Er-Miao-San ameliorated microalbuminuria and renal histopathology, as well as decreased XOD activity in hyperuricemic rats. (iii) Combination of Si-Wu-Tang and Er-Miao-San also led to a greater increase in OAT1 and OAT3 expression than did Si-Wu-Tang alone. These results clearly demonstrated the combined protective effects of Si-Wu-Tang and Er-Miao-San on hyperuricemia and renal impairment in rats, and then provided a novel therapeutic approach for the treatment of uric acid nephropathy.

This study demonstrated that treatment with Si-Wu-Tang plus Er-Miao-San for 4 weeks significantly decreased the serum uric acid, creatinine and BUN levels levels in hyperuricemic rats. Moreover, Si-Wu-Tang plus Er-Miao-San was more effective than Si-Wu-Tang alone. 
Thus, the combined use of the two Chinese herbal formulas (Si-Wu-Tang plus Er-Miao-San) produced mutual enhancement in ameliorating hyperuricemia and renal impairment in rats.

It was reported that supplementation with adenine induced hyperuricemia and nephropathy in rats [17]. Previous studies reported that the uricase inhibitor potassium oxonate was used to induce hyperuricemia in rats $[18,19]$. Therefore, adenine and potassium oxonate were used to induce hyperuricemia in this study and we found that adenine and potassium oxonate administration resulted in elevated levels of serum uric acid, creatinine and BUN. These results indicated the adenine and potassium oxonate-induced hyperuricemia rat model was successful.

Previus studies demonstrated that xanthine dehydrogenase (XDH) was a cytoplasmic enzyme implicated in hydroxylation of hypoxanthine to xanthine and its oxidation to uric acid and a relevant source of oxidants in vasculature [20-22]. XDH might undergo limited proteolysis or oxidation of crucial cysteine residues to form the xanthine oxidase (XOD). It has been reported that the traditional Chinese herbal formula $\mathrm{Si}-\mathrm{Wu}$-Tang prevents oxidative damage by activating Nrf2-mediated detoxifying/antioxidant genes [23]. Cortex Phellodendri Chinensis and Atractylodes Lancea, the ingredients of Er-Miao-San were reported to have antioxidant and anti-inflammatory activities [24-27]. The recent study demonstrated that Er-Miao-Wan reduced serum uric acid level and inhibited XDH and XO activities in oxonate-induced hyperuricemia model [14]. In this study, hyperuricemic rats showed a significantly increased in serum XOD activity and serum uric acid level, which was reversed by Si-Wu-Tang plus Er-Miao-San. Therefore, the inhibited effects of Si-Wu-Tang plus Er-Miao-San on serum XOD avtivity were likely to be accountable for its hypouricemic action. Moreover, Si-Wu-Tang plus Er-Miao-San ameliorated microalbuminuria and renal histopathology, as well as decreased serum TCH concentration in hyperuricemic rats. These results clearly demonstrated that Si-Wu-Tang plus Er-Miao-San significantly decreased the serum XOD activity and uric acid level, thus ameliorated structural and functional abnormalities in hyperuricemic rats.

To reveal the mechanisms underlying the hypouricemic action of Si-Wu-Tang plus Er-Miao-San, we investigated the effects Si-Wu-Tang plus Er-Miao-San on OAT1 and OAT3 expression in hyperuricemic rats. Tubular transporters were important for handling of uric acid in renal tubular [28]. Organic anion transporter 1 (OAT1, SLC22A6) and OAT3 (SLC22A8), distributed at the basolateral membrane, were responsible for urate uptake from the blood across the basolateral membrane into proximal tubule cells [29]. The mRNA and protein expression levels of basolateral organic anion transporters OAT1 and OAT3 significantly decreased in hyperuricemic rats [30]. In this study, the hyperuricemic rats also showed reduced protein expression of OAT1 and OAT3 detected by western blot. These observations suggest that OAT1 and OAT3 may play important roles in the impaired urate excretion and hyperuricemia. However, combination of Si-Wu-Tang and Er-Miao-San led to a greater increase in OAT1 and OAT3 expression in hyperuricemic rats than did Siwutang alone. These results clearly demonstrated that the hypouricemic effects of Si-Wu-Tang plus Er-Miao-San were partly through upregulation of OAT1 and OAT3 expression.

In this study the doses of Si-Wu-Tang and Si-Wu-Tang plus Er-Miao-San were calculated by the formula that converts dosage of human into that of rat in terms of the respective body surface areas according to the Chinese Medicine Pharmacology Research Technology (1994). Thus, the doses of Si-Wu-Tang at $3.78 \mathrm{~g}$ raw materials/kg/day and Si-Wu-Tang plus Er-Miao-San at $6.48 \mathrm{~g}$ raw materials/kg/day were chosen in our study.

Since the combination of potassium oxonate with adenine was previously described in literature to induce the experimental model of uric acid nephropathy [31], we also used this model to investigate the synergistically proctective effects of Si-Wu-Tang and Er-Miao-San on hyperuricemia and renal impairment in rats. Moreover, adenine that is successfully used for hyperuricemia induction enters unmetabolized into the portal vasculature of the rat and therefore is metabolized in the liver [32] that will determine its impact on uricemia. Previous study demonstrated that modified Siwu Siteng Decoction inhibited the liver xanthine oxidase (XO) activity and decreased serum uric acid in rats induced by hypoxanthine and oxonic

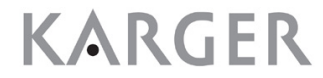


acid potassium salt [33]. Clinical study further indicated that Siwu Decoction attenuated hyperuricemia and renal impairment in patients with Type 2 diabetes [34]. Thus, we will further examine the influence of Si-Wu-Tang or Si-Wu-Tang plus Er-Miao-San on liver XO activity in hyperuricemic rats in future study.

Finally, we discussed the possible active components of the Si-Wu-Tang, Si-Wu-Tang plus Er-Miao-San and their influence on certain mechanisms of oxidative tissue injury relevant to the model used. Previous study found that the level of reactive oxygen species was higher in the hyperuricemic rats and Smilacis Glabrae Rhizoma could ameliorate the oxidative stress caused by hyperuricemia through upregulating catalase expression [35]. There were nine compounds including Phellodendrine, Magnoflorine, Lotusine, Menisperine, Palmatine, Columbamine, Jatrorrhizine, Berberrubine and Berberine which were screened and identified as potential XO inhibitors in Ermiaowan. Among of them, Palmatine, Berberrubine and Berberine had better binding ability to XO [36]. A recent study reported that Er-MiaoSan inhibited inflammatory mediators and iNOS expression in LPS-stimulated RAW264.7 macrophages via the suppression of NF- $\kappa B$ pathway and MAPKs activation [37]. Previous study reported that z-liguistilide was discovered as the main biologically active component of $\mathrm{Si}$-Wu-Tang responsible for activating the Nrf2 pathway [23]. Moreover, recent study showed that the alkaloids components of Cortex Phellodendri Chinensis were the biologically active substances for protecting HUVEC induced by urate in Ermiao Wan categorized formula [38]. It has been demonstrated that the alcohol soluble ingredients of astragalus membranaceus and Siwu decoction in modified Siwu Siteng Decoction can effectively reduce the level of blood uric acid in hyperuricemic rats [33].

In conclusion, this study clearly demonstrated that Si-Wu-Tang plus Er-MiaoSan, which are used in traditional Chinese formulas to treat gout and hyperuricemia, ameliorated hyperuricemia and renal impairment in rats through upregulation of OAT1 and OAT3 expression. This novel finding will provide an alternative therapy for treatment of hyperuricemia and renal dysfunction in metabolic syndrome.

\section{Acknowledgments}

This work was supported by grants from National Natural Science Foundation of China (81000305, 81573738 to Dingkun Gui and 81270824 to Niansong Wang) and Health Science and Technology of Social Development Bureau, Pudong New Area, Shanghai (PW2009A-21 to Yongping Guo). This work was funded by grants from Traditional Chinese Medicine Foundation of Shanghai Municipal Commission of Health and Family Planning (2014JP018A), Joint Research Project of Pudong Health Bureau of Shanghai (PW2013D-5), Pudong New Area Science and Technology Development Fund (PKJ2014-Y09) and Shanghai Municipal Commission of Health and Family Planning Research Project (201440309). This work was also supported by grants from Key Disciplines Group Construction Project of Pudong Health Bureau of Shanghai (PWZxq2014-07), Medical-Engineering Cross Project of Shanghai Jiao Tong University (YG2014MS20) and Outstanding Young Talents Program in East campus, Shanghai Jiao Tong University Affiliated Sixth People's Hospital. This work was also supported by the National Key Technology Research and Development Program of China (2013BAI02B00, 2013BAI02B04) and the Special Scientific Research in the Chinese Medicine Industry of the Ministry of Science and Technology (201007005).

\section{Disclosure Statement}

The authors have declared that no competing interests exist. 


\section{Cellular Physiology Cell Physiol Biochem 2015;37:1491-1502 \begin{tabular}{ll|l} 
and Biochemistry & $\begin{array}{l}\text { DOI: 10.1159/000438517 } \\
\text { Published online: October 29, 2015 }\end{array}$ & $\begin{array}{l}\text { ( ) } 2015 \text { S. Karger AG, Basel } \\
\text { www.karger.com/cpb }\end{array}$ \\
\hline
\end{tabular}

\section{References}

1 Zhang L, Wang F, Wang L, Wang W, Liu B, Liu J, Chen M, He Q Liao Y, Yu X, Chen N, Zhang JE, Hu Z, Liu F, Hong D, Ma L, Liu H, Zhou X, Chen J, Pan L, Chen W, Wang W, Li X, Wang H: Prevalence of chronic kidney disease in China: a cross-sectional survey. Lancet 2012;379:815-822.

2 Lin B, Shao L, Luo Q Ou-Yang L, Zhou F, Du B, He Q Wu J, Xu N, Chen J: Prevalence of chronic kidney disease and its association with metabolic diseases: a cross-sectional survey in Zhejiang province, Eastern China. BMC Nephrol 2014;15:36.

3 Sedaghat S, Hoorn EJ, van Rooij FJ, Hofman A, Franco OH, Witteman JC, Dehghan A: Serum uric acid and chronic kidney disease: the role of hypertension. PLoS One 2013;18:e76827.

4 Storhaug HM, Norvik JV, Toft I, Eriksen BO, Løchen ML, Zykova S, Solbu M, White S, Chadban S, Jenssen T: Uric acid is a risk factor for ischemic stroke and all-cause mortality in the general population: a gender specific analysis from The Troms $\varnothing$ Study. BMC Cardiovasc Disord 2013;13:115.

5 Chen JH, Chuang SY, Chen HJ, Yeh WT, Pan WH: Serum uric acid level as an independent risk factor for all-cause, cardiovascular, and ischemic stroke mortality: a Chinese cohort study. Arthritis Rheum 2009;61:225-232.

6 Chen IH, Kuo MC, Hwang SJ, Chang JM, Chen HC: Allopurinol-induced severe hypersensitivity with acute renal failure. Kaohsiung J Med Sci 2005;21:228-232.

7 Horiuchi H, Ota M, Nishimura S, Kaneko H., Kasahara Y, Ohta T, Komoriya K: Allopurinol induces renal toxicity by impairing pyrimidine metabolism in mice. Life Sci 2000;66:2051-2070.

8 Mari E, Ricci F, Imberti D, Gallerani M: Agranulocytosis: an adverse effect of allopurinol treatment. Italian J Med 2011;5:120-123.

9 Pluim HJ, Deuren MV, Wetzels JFM: The allopurinol hypersensitivity syndrome. Neth J Med 1998;52:107110.

10 Ahmad NS, FarmanM, Najmi MH, Mian KB, Hasan A: Pharmacological basis for use of Pistacia integerrima leaves in hyperuricemia and gout. J Ethnopharmacol 2008;117:478-482.

11 An J, Yang HJ, Park K, Lee J, Kim BW: Reparatory and preventive effects of oriental herb extract mixture (OHEM) on hyperuricemia and gout. Food Sci Biotechnol 2010;19:517-524.

12 Liang QD , Lu XQ, Ma ZC, Tan HL, Ma BP , Gao Y, Wang SQ: Preliminary study on hematopoietic constituents of Si-Wu-Tang. Zhongguo Zhong Yao Za Zhi 2004;29:546-549.

13 Dai Y, But PP, Chan YP, Matsuda H, Kubo M: Antipruritic and antiinflammatory effects of aqueous extract from Si-Wu-Tang. Biol Pharm Bull 2002;25:1175-1178.

14 Kong LD, Yang C, Ge F, Wang HD, Guo YS: A Chinese herbal medicine Ermiao wan reduces serum uric acid level and inhibits liver xanthine dehydrogenase and xanthine oxidase in mice. J Ethnopharmacol 2004;93:325-330.

15 Kramer HM, Curhan G: The association between gout and nephrolithiasis: the National Health and Nutrition Examination Survey III, 1988-1994. Am J Kidney Dis 2002;40:37-42.

16 Li Y, Stamler J, Xiao Z, Folsom A, Tao S, Zhang, H: Serum uric acid and its correlates in Chinese adult populations, urban and rural, of Beijing: the PRC-USA collaborative study in cardiovascular and cardiopulmonary epidemiology. Int J Epidemiol 1997;26:288-296.

17 Brulé D, Sarwar G, Savoie L, Campbell J, Van Zeggelaar M: Differences in uricogenic effects of dietary purine bases, nucleosides and nucleotides in rats. J Nutr 1988;118:780-786.

18 Hou SX, Zhu WJ, Pang MQ, Jeffry J, Zhou LL: Protective effect of iridoid glycosides from Paederia scandens (LOUR.) MERRILL (Rubiaceae) on uric acid nephropathy rats induced by yeast and potassium oxonate. Food Chem Toxicol 2014;64:57-64.

19 Yan H, Ma Y, Liu M, Zhou L: The dual actions of Paederia scandens extract as a hypouricemic agent: xanthine oxidase inhibitory activity and uricosuric effect. Planta Med 2008;74:1345-1350.

20 Gibbings S, Elkins ND, Fitzgerald H, Tiao J, Weyman ME, Shibao G, Fini MA, Wright RM: Xanthine oxidoreductase promotes the inflammatory state of mononuclear phagocytes through effects on chemokine expression, peroxisome proliferator-activated receptor- $\gamma$ sumoylation, and HIF- $1 \alpha$. J Biol Chem 2011;286:961-975.

21 Pacher P, Nivorozhkin A, Szabó C: Therapeutic effects of xanthine oxidase inhibitors: renaissance half a century after the discovery of allopurinol. Pharmacol Rev 2006;58:87-114. 


\section{Cellular Physiology Cell Physiol Biochem 2015;37:1491-1502

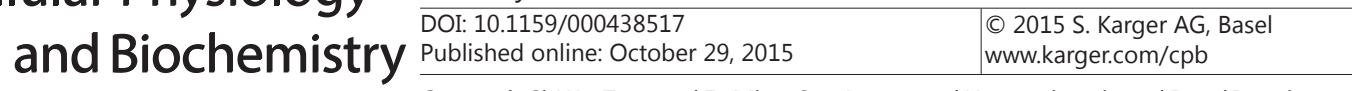

22 Houston M, Estevez A, Chumley P, Aslan M, Marklund S, Parks DA, Freeman BA: Binding of xanthine oxidase to vascular endothelium: kinetic characterization and oxidative impairment of nitric oxide-dependent signaling. J Biol Chem 1999;274:4985-4994.

23 Liu M, Ravula R, Wang Z, Zuo Z, Chow MS, Thakkar A, Prabhu S, Andresen B, Huang Y: Traditional Chinese medicinal formula Si-Wu-Tang prevents oxidative damage by activating Nrf2-mediated detoxifying/ antioxidant genes. Cell Biosci 2014;4:8.

24 Endo K, Taguchi T, Taguchi F, Hikino H, Yamahara J, Fujimura H: Anti-inflammatory principles of Atractylodes rhizomes. Chem Pharm Bull (Tokyo) 1979;27:2954-2958.

25 Yamahara J, Matsuda H, Naitoh Y, Fujimura H, Tamai Y: Antianoxic action and active constituents of atractylodis lanceae rhizoma. Chem Pharm Bull (Tokyo) 1990;38:2033-2034.

26 Resch M, Steigel A, Chen ZL, Bauer R: 5-Lipoxygenase and cyclooxygenase-1 inhibitory active compounds from Atractylodes lancea. J Nat Prod 1998; 61:347-350.

27 Lee YH, Kim H, Hong EK, Kang BH, Kim SJ: Water extract of 1:1 mixture of phellodendron cortex and aralia cortex has inhibitory effects on oxidative stress in kidney of diabetic rats. J Ethnopharmacol 2000;73:429436.

28 Bobulescu IA, Moe OW: Renal transport of uric acid: evolving concepts and uncertainties. Adv Chronic Kidney Dis 2012;19:358-371.

29 Eraly SA, Vallon V, Rieg T, Gangoiti JA, Wikoff WR, Siuzdak G, Barshop BA,Nigam SK: Multiple organic anion transporters contribute to net renal excretion of uric acid. Physiol Genomics 2008;33:180-192.

30 Habu Y, Yano I, Takeuchi A, Saito H, Okuda M, Fukatsu A, Inui K: Decreased activity of basolateral organic ion transports in hyperuricemic rat kidney: roles of organic I on transporters, rOAT1, rOAT3 and rOCT2. Biochem Pharmacol 2003;66:1107-1114.

31 Zhu W, Pang M, Dong L, Huang X, Wang S, Zhou L: Anti-inflammatory and immunomodulatory effects of iridoid glycosides from Paederia scandens (LOUR.) MERRILL (Rubiaceae) on uric acid nephropathy rats. Life Sci 2012;91:369-376.

32 Salati LM, Gross CJ, Henderson LM, Savaiano DA: Absorption and metabolism of adenine, adenosine-5'monophosphate, adenosine and hypoxanthine by the isolated vascularly perfused rat small intestine. J Nutr 1984;114:753-760.

33 Yang X, Gao K, Wang Y, Zhang Y, Guo H, Su H: Effects of Different Extracts of Modified Siwu Siteng Decoction on Uric Acid and Xanthine Oxidase in Rats with Hyperuricemia. Chin J Inform Traditional Chin Med 2014;21:66-68.

34 Hu Y, Guo C, Li J, Wang B, Wu S: Supplemented Siwu Decoction for type 2 diabetes with asymptomatic hyperuricemia: a clinical observation of 38 cases. J Tradit Chin Med 2007;48:515-517.

35 Hong Q, Yu S, Mei Y, Lv Y, Chen D, Wang Y, Geng W, Wu D, Cai G, Chen X: Smilacis Glabrae Rhizoma reduces oxidative stress caused by hyperuricemia via up regulation of catalase. Cell Physiol Biochem 2014;34:1675-1685.

36 Xu C, Liu S, Liu Z, Song F: Screening of Xanthine Oxidase Inhibitors in Traditional Chinese Herbal Formulae Ermiaowan Using Ultrafiltration UPLC-MS. Chem J Chinese Univ 2014, 35:1640-1645.

37 Chen G, Li KK, Fung CH, Liu CL, Wong HL, Leung PC, Ko CH: Er-Miao-San, a traditional herbal formula containing Rhizoma Atractylodis and Cortex Phellodendri inhibits inflammatory mediators in LPSstimulated RAW264.7 macrophages through inhibition of NF- $\kappa$ B pathway and MAPKs activation. J Ethnopharmacol 2014;154:711-718.

38 Yin L, Deng H, Zhu X, Li X, Duan J, Ding A: Study on role for protecting HUVEC damaged by urate and content variation of alkaloids components in Ermiao Wan categorized formula. Chem J Chinese Univ 2009;30:1522-1527. 